\title{
Different diagnostic performance of plasma fibrinogen and D-dimer in periprosthetic joint infection: a propensity score matched study
}

Xi Chen, Wenwei Qian*, Xisheng Weng, Jin Lin, Jin Jin, Yiou Wang and Shibai Zhu

\begin{abstract}
Background: Fibrinogen (Fbg) and D-dimer have been used as biomarkers for the diagnosis of periprosthetic joint infection (PJI). However, previous research has reported conflicting results on the diagnostic value of D-dimer in comparison to Fbg, C-reactive protein (CRP), and erythrocyte sedimentation rate (ESR).

Aim: This study aimed to: (1) determine the optimal threshold of plasma Fbg and D-dimer in the diagnosis of PJI and compare their diagnostic value to that of CRP and ESR; and (2) investigate whether Fbg and D-dimer perform differently than CRP and ESR as diagnostic indicators for different types of PJI.

Methods: A total of 115 revision cases after total hip arthroplasty (THA) and total knee arthroplasty (TKA) were identified. Based on demographic characteristics, 25 culture-positive cases were matched to 50 culture-negative cases using propensity score matching. Sensitivity, specificity, receiver operating characteristics (ROC), negative predictive value (NPV), and positive predictive value (PPV) were calculated and compared.
\end{abstract}

Results: The optimal thresholds were $2.72 \mathrm{mg} / \mathrm{L}$ for D-dimer, $3.655 \mathrm{~g} / \mathrm{L}$ for Fbg, $12.64 \mathrm{mg} / \mathrm{L}$ for CRP, and $27 \mathrm{~mm} / \mathrm{h}$ for ESR. Levels of plasma Fbg, D-dimer, CRP, and ESR were significantly higher in the culture-positive group than the culture-negative group. Fbg, D-dimer, CRP, and ESR showed sensitivity of 0.92, 0.56, 0.92, and 0.88, respectively, and showed specificity of $0.84,0.96,0.94$, and 0.80 , respectively. The ROC curve showed that CRP has the highest area under the curve (AUC) (0.94), followed by Fbg (0.90), ESR (0.87), and D-dimer (0.81).

Conclusions: Plasma Fbg exhibited a similar diagnostic performance compared to CRP and ESR in predicting culture-positive results in PJI. Plasma D-dimer showed high specificity but low sensitivity. In our study, Fbg and D-dimer did not show better diagnostic performance with different pathogens and different types of PJI. Further studies are required to investigate the difference between serum D-dimer and plasma D-dimer in the arthroplasty population.

Keywords: Periprosthetic joint infection, Fibrinogen, D-dimer

\footnotetext{
* Correspondence: qianww007@163.com

Department of Orthopedic Surgery, Peking Union Medical College Hospital, Peking Union Medical College, Chinese Academy of Medical Science, Beijing, China
}

C C The Author(s). 2021 Open Access This article is licensed under a Creative Commons Attribution 4.0 International License, which permits use, sharing, adaptation, distribution and reproduction in any medium or format, as long as you give appropriate credit to the original author(s) and the source, provide a link to the Creative Commons licence, and indicate if changes were made. The images or other third party material in this article are included in the article's Creative Commons licence, unless indicated otherwise in a credit line to the material. If material is not included in the article's Creative Commons licence and your intended use is not permitted by statutory regulation or exceeds the permitted use, you will need to obtain permission directly from the copyright holder. To view a copy of this licence, visit http://creativecommons.org/licenses/by/4.0/. The Creative Commons Public Domain Dedication waiver (http://creativecommons.org/publicdomain/zero/1.0/) applies to the data made available in this article, unless otherwise stated in a credit line to the data. 


\section{Article summary Article focus}

1. Determine the optimal threshold of plasma Fbg, Ddimer, CRP, and ESR in the diagnosis of culturepositive PJI in revision arthroplasty.

2. Compare the diagnostic value of Fbg and D-dimer to that of CRP and ESR in PJI and explore potential causes for any observed differences.

3. Investigate whether Fbg and D-dimer perform differently than CRP and ESR in diagnosing different types of culture-positive PJI and associated pathogens.

\section{Key messages}

1. Plasma Fbg exhibited similar diagnostic performance in PJI compared to CRP and ESR.

2. Plasma D-dimer is of limited diagnostic value in PJI.

3. Further studies are required to investigate the differences in diagnostic performance between serum D-dimer and plasma D-dimer in the arthroplasty population.

\section{Strengths/Limitations}

1. Propensity score matching was utilized to eliminate bias caused by confounding factors (age, sex, BMI, and comorbidity). Another benefit of matching is to gain more efficiency and reduce bias in studies with a small sample size.

2. Sample size was limited in our study.

3. There were significantly more knee cases in the PJI group than in the Aseptic group, which may have led to potential bias.

\section{Background}

The diagnosis of periprosthetic joint infection (PJI) has been challenging for decades. Current diagnostic criteria include physical findings, culture, histological analysis, and serological/synovial fluid biomarkers $[1,2]$. However, physical signs are sometimes subtle in patients with PJI. Histological analysis is unavailable until after surgery. Leukocyte count and other markers from synovial fluid have good diagnostic value. However, it can be difficult to acquire a sufficient amount of joint fluid for a culture or other tests [3], and venous blood samples are more easily accessible. Erythrocyte sedimentation rate (ESR) and C-reactive protein (CRP) are essential serological markers in the diagnosis of PJI. However, ESR and CRP values can be normal in cases where PJI is caused by a low-virulence organism $[4,5]$. Therefore, the use of more accessible biomarkers would improve efficiency and accuracy in the diagnosis of PJI, especially if they exhibit good diagnostic performance in chronic and low-virulence infections.

Novel biomarkers including D-dimer, fibrinogen (Fbg), alpha-defensin, and interleukin-6 (IL-6) have been introduced to improve the diagnostic criteria of PJI [6]. Ddimer and Fbg are routinely tested in most medical facilities. Parvizi et al. [1] introduced D-dimer in the 2018 Musculoskeletal Infection Society (MSIS) diagnostic criteria, in which it plays an equal role to that of CRP. However, there are conflicting research results on the diagnostic value of $\mathrm{D}$-dimer in comparison to $\mathrm{Fbg}$, CRP, and ESR, in part because different optimal diagnostic thresholds have been utilized [3, 7-9]. Shahi et al. [3] reported a high diagnostic value for D-dimer. Li et al. [8] reported that $\mathrm{D}$-dimer has limited diagnostic value in PJI, while Fbg showed promising diagnostic value. Limited evidence has been found on whether these biomarkers are valuable in recognizing infections caused by low-virulence pathogens and chronic infections.

Therefore, this study aims to: (1) determine the optimal threshold of plasma Fbg and D-dimer in the diagnosis of PJI and compare their diagnostic value to that of CRP and ESR; and (2) investigate whether Fbg and D-dimer perform differently than CRP and ESR as diagnostic indicators for different types of PJI.

\section{Methods}

Approval from the Institutional Ethics Committee was obtained. We retrospectively reviewed patient admission records from December 2012 to March 2020. Patients admitted for total knee arthroplasty revision and total hip arthroplasty revision were recorded. Patients were excluded if they: (1) underwent revision surgery for dislocation and acute periprosthetic fracture; or (2) underwent second stage revision (reimplantation) surgery following PJI. Patients were then divided into culture-positive cases and culture-negative cases based on culture results.

Propensity score matching was used to match each culture-positive case to culture-negative cases. The following covariates were matched: (1) age; (2) sex; (3) height; (4) weight; (5) BMI; and (6) comorbidities, including coagulation disorder, auto-immune disease and malignancy, or taking anticoagulation medication or immunosuppressants. Four culture-positive cases were excluded due to unsuccessful matching. Eventually, 25 culture positive cases were matched to 50 culturenegative cases.

Blood samples were taken 1-3 days following admission and prior to the day of surgery. Plasma D-dimer, Fbg, CRP, and ESR were tested among a series of preoperative lab draws. Pre-operative joint aspirations were taken when PJI was suspected. Results of physical examination, culture, and time of PJI symptomatic onset were 
recorded. All cases were followed up at three to six months post-operatively in outpatient clinic.

Statistical analyses were conducted with SPSS 25. Propensity score matching was conducted using STATA 14. The ROC curve was generated by Graphpad 8.0. Continuous variables were analyzed by unpaired $t$ test and results were recorded as means and standard deviations. Dichotomous variables were analyzed by chi-square test and recorded as frequencies and ratios. A P-value less than 0.05 was considered statistically significant. The sensitivity, specificity, positive predictive value (PPV), and negative predictive value (NPV) of each biomarker were calculated and recorded with $95 \%$ confidence intervals $(95 \% \mathrm{CI})$. Positive predictive value is the probability that a patient with a positive test result has the disease. Negative predictive value is the probability that a patient with a negative test result does not have the disease. The Youden index was used to determine the optimal threshold for D-dimer and Fbg. A receiver operating characteristics (ROC) curve was generated, and we used its corresponding area under the curve (AUC) and $95 \% \mathrm{CI}$ to determine the diagnostic value of each biomarker.

\section{Results}

The demographic characteristics of included cases are listed in Table 1. There was no statistical difference in terms of sex, age, BMI, and comorbidities between the two groups. There was a significantly higher proportion of TKA cases in the culture-positive group than in the culture-negative group. The levels of plasma D-dimer, Fbg, CRP, and ESR were significantly higher in the culture-positive group than in the culture-negative group (Table 1).
The optimal threshold of D-dimer and Fbg was determined based on the Youden Index. The optimal thresholds were determined to be $2.72 \mathrm{mg} / \mathrm{L}$ for D-dimer and $3.655 \mathrm{~g} / \mathrm{L}$ for Fbg. The ROC curve showed that CRP had the highest AUC (0.94), followed by Fbg (0.90), ESR (0.87), and D-dimer (0.) (Fig. 1). CRP and Fbg had the highest sensitivity of 0.92 , followed by ESR $(0.88)$. The sensitivity of D-dimer was 0.56 . D-dimer showed the highest specificity (0.96), followed by CRP (0.94), Fbg (0.84), and ESR (0.80). The positive predictive value for CRP, Fbg, ESR, and D-dimer were 0.89, 0.75, 0.69, and 0.74 , respectively. The negative predictive value for CRP, Fbg, ESR, and D-dimer were 0.96, 0.91, 0.93, and 0.95, respectively. The diagnostic performance of $\mathrm{Fbg}, \mathrm{D}$ dimer, CRP, and ESR is listed in Table 2.

Nine cases were defined as acute PJI (diagnosis within 3 months after primary implantation) and they were culture-positive. Sixteen cases were defined as chronic PJI. For acute PJI, the positive rates of Fbg, D-dimer, CRP, and ESR were $88.9 \%, 66.7 \%, 100 \%$, and $88.9 \%$, respectively. For chronic PJI, the positive rates of Fbg, Ddimer, CRP, and ESR were $93.8 \%, 50 \%, 87.5 \%$, and $87.5 \%$, respectively. Coagulase-negative Staphylococcus was identified in nine cases and coagulase-positive Staphylococcus was identified in four cases. The types of infection and the responsible pathogens are listed in Table 3.

\section{Discussion}

Fibrinogen and D-dimer are by-products of the process of fibrin clot breakdown. Increased levels of both Fbg and D-dimer represent the activation of the coagulation process [10]. Researchers have examined the relationship between the processes of coagulation and inflammation.

Table 1 Comparison of demographic characteristics and biomarkers between groups

\begin{tabular}{|c|c|c|c|}
\hline & Culture-positive $(n=25)$ & Culture-negative $(n=50)$ & $P$-Value \\
\hline Age (yrs) & $60.88 \pm 14.21$ & $65.24 \pm 9.71$ & 0.176 \\
\hline Sex & & & 0.307 \\
\hline Male & 10 & 14 & \\
\hline Female & 15 & 36 & \\
\hline BMI & $26.15 \pm 4.08$ & $25.17 \pm 3.83$ & 0.325 \\
\hline Hip/Knee & & & 0.007 \\
\hline Hip & 6 & 29 & \\
\hline Knee & 19 & 21 & \\
\hline Comorbidity $^{a}$ & 10 & 14 & 0.307 \\
\hline D-dimer (mg/L) & $3.96 \pm 3.14$ & $1.19 \pm 0.91$ & $<0.001$ \\
\hline $\mathrm{Fbg}(\mathrm{g} / \mathrm{L})$ & $4.75 \pm 1.34$ & $3.08 \pm 0.60$ & $<0.001$ \\
\hline CRP (mg/L) & $61.31+63.93$ & $5.09 \pm 5.95$ & $<0.001$ \\
\hline ESR $(\mathrm{mm} / \mathrm{h})$ & $56.88 \pm 31.89$ & $19.70 \pm 16.58$ & $<0.001$ \\
\hline
\end{tabular}

Comorbidity $^{\mathrm{a}}$ Patients with coagulation disorder, autoimmune disease, other infections or malignancy, or those taking anti-coagulation medication or immunosuppressors 


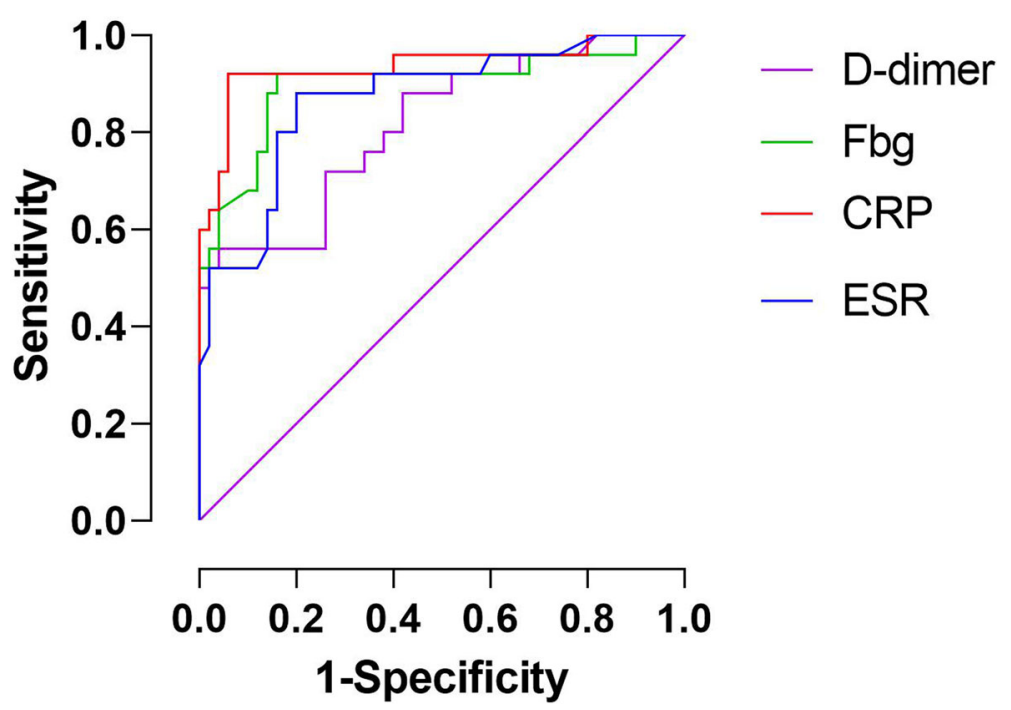

Fig. 1 ROC Curve

Coagulation biomarkers have pro-inflammatory effects, as fibrin mediates the inflammatory process and Ddimer promotes neutrophil and monocyte activation. Persistent inflammation also contributes to hypercoagulable state $[11,12]$.

To date, most studies that have focused on the diagnostic value of D-dimer and Fbg have utilized the MSIS criteria [9, 13-15]. However, the MSIS criteria have been called into question by researchers and is susceptible to subjectivity among clinicians, which could introduce selection bias. Therefore, culture result was used as the primary objective outcome measurement in this study. This allowed us to analyze CRP and ESR as well since they are not interpreted as part of a gold standard test for PJI.

Based on the AUC of each biomarker, Fbg (0.90), CRP (0.93), and ESR (0.87) exhibited similar diagnostic value, while D-dimer (0.81) exhibited a comparatively poor diagnostic value. The sensitivity of D-dimer was much lower than that of Fbg, CRP, and ESR. Previous researchers have reported conflicting results on the role of D-dimer in the diagnosis of PJI. A meta-analysis published in 2020 by Liu et al. [16] found good diagnostic performance of Fbg and moderate diagnostic performance of D-dimer. Our previous systematic review concluded that serum D-dimer is of comparable diagnostic value to CRP and ESR for PJI, while in this series, plasma D-dimer was of limited diagnostic value [17]. One explanation for these differences is that serum and plasma D-dimer exhibit different diagnostic performance. In serum samples, D-dimer is measured after standardized coagulation. Cross-linked fibrin degradation products appear in the blood before standardized coagulation and are mostly included in serum samples after coagulation. The fibrin degradation products remaining in the serum sample adds to the measured serum Ddimer, which may result in a higher level of serum Ddimer comparing to plasma D-dimer. However, the effect of residual fibrin degradation products in the serum has not been quantified [18-20]. Paniccia et al. [18] found serum D-dimer was significantly higher than plasma D-dimer in the majority of pregnant women and they found no correlation between serum D-dimer and plasma D-dimer. To our knowledge, there is no study reporting the difference between serum and plasma Ddimer in arthroplasty populations. Contemporary researchers have reported on the limited diagnostic value of plasma D-dimer and conflicting results regarding Ddimer. Two studies $[8,15]$ have shown the limited diagnostic value of plasma D-dimer compared to CRP and

Table 2 Diagnostic performance of each biomarker

\begin{tabular}{lllllll}
\hline & Threshold & Sensitivity & Specificity & AUC (95 \% Cl) & PPV & NPV \\
\hline Fbg $(\mathrm{g} / \mathrm{L})$ & 3.655 & 0.92 & 0.84 & $0.90(0.808-0.987)$ & 0.758 & 0.912 \\
D-dimer $(\mathrm{mg} / \mathrm{L})$ & 2.72 & 0.56 & 0.96 & $0.81(0.710-0.919)$ & 0.741 & 0.955 \\
CRP $(\mathrm{mg} / \mathrm{L})$ & 12.64 & 0.92 & 0.94 & $0.93(0.866-0.999)$ & 0.885 & 0.959 \\
ESR $(\mathrm{mm} / \mathrm{h})$ & 27 & 0.88 & 0.80 & $0.87(0.781-0.957)$ & 0.687 & 0.930 \\
\hline
\end{tabular}

PPV positive predictive value, NPV negative predictive value 
Table 3 Types of PJI and identified pathogens

\begin{tabular}{llll}
\hline & Acute $(\boldsymbol{n}=\mathbf{9})$ & Chronic $(\boldsymbol{n}=\mathbf{1 6})$ & Total $(\boldsymbol{n}=\mathbf{2 5})$ \\
\hline Positive culture & 9 & 16 & 25 \\
Coagulase-negative Staphylococcus & & 8 & 8 \\
Staphylococcus epidermidis & 0 & 0 & 1 \\
Staphylococcus capitis & 1 & & 4 \\
Coagulase-positive Staphylococcus & & 2 & 1 \\
Staphylococcus aureus & 2 & 1 & 1 \\
Streptococcus & & 0 & 1 \\
Streptococcus mitis & 0 & 0 & 1 \\
Streptococcus pneumoniae & 1 & 1 & 3 \\
Streptococcus agalactiae & 1 & 2 & 1 \\
Streptococcus anginosus & 0 & 0 & 1 \\
Escherichia coli & 1 & 0 & 1 \\
Brucella & 1 & 1 & 1 \\
Salmonella & 1 & 1 & 1 \\
Klebsiella pneumoniae & 0 & 0 & 1 \\
Bacillus fragilis & 1 & 1 \\
Corynebacterium rhizogenes & 0 & 1 & \\
\hline
\end{tabular}

ESR. Shahi et al. [3] reported promising results of serum D-dimer, while Pannu et al. [21] and Huang et al. [7] reported limited diagnostic value of serum D-dimer. Differing testing methods and samples were used in different hospitals, which may have influenced our clinical interpretation of these studies. As reported by Liu et al. [16], multiple methods can be used to assess fibrinogen and other biomarkers. Further studies in this field are required to investigate the diagnostic value of these biomarkers with different testing methods and samples.

Racial difference is considered one of the reasons for the differential diagnostic value of D-dimer across different studies. However, based on the data from previous research, studies published in the United States of America reported controversial outcomes on the diagnostic value of D-dimer $[3,21]$, as did studies published in China $[7,8,14,15]$.

Plasma Fbg showed comparable diagnostic performance in PJI compared to CRP and ESR. Our results are inconsistent with the results from $\mathrm{Li}$ et al. [8]. Fibrinogen has been found to play several key roles in host antimicrobial defense. Fibrinogen can limit the growth and dissemination of bacteria within infected tissue and can support the activation of host immune cells [22]. In peritoneal infection, fibrinogen can contain Staphylococcus aureus and other pathogens [23].

As a coagulation biomarker, Fbg is superior to Ddimer in the diagnosis of PJI. One possible explanation might be that Fbg and D-dimer are associated with the infection process by different mechanisms. Another reason might be that $\mathrm{D}$-dimer has shown good diagnostic value in detecting deep vein thrombosis, pulmonary embolism and other inflammatory diseases [24]. However, limited evidence has been reported regarding the diagnostic performance of Fbg in these conditions [25]. Patients with thrombosis and inflammatory diseases were included in our study, which may have influenced the diagnostic value of D-dimer. However, further studies are required to explore the rationale behind the differential diagnostic performance of D-dimer and Fbg.

Fbg and D-dimer did not exhibit better diagnostic performance than CRP and ESR in chronic PJI. Among the 16 cases with chronic PJI in our series, Fbg was positive in 15 cases. D-dimer was positive in 8 cases, CRP was positive in 14 cases, and ESR was positive in 14 cases. Coagulase-negative Staphylococcus was identified in nine cases, Fbg was positive in all cases, D-dimer was positive in five cases and ESR and CRP were positive in eight cases. Unlike the findings in our series, Shahi et al. [3] reported that D-dimer was positive in 17 of 19 PJI cases with negative-culture results, thus performing better than CRP and ESR.

Patients' comorbidities and demographic characteristics could have influenced the results of each biomarker. Comorbidities that may have influenced the interpretation of the index test in our study were matched between the culture-positive group and the culturenegative group by propensity scores to avoid bias caused by the uneven distribution of confounding factors. The inclusion of patients with comorbidities provided realworld data that are more relevant to clinical practice and is supported by a previous study [15]. However, it should 
be noted that the inclusion of these patients may have influenced our interpretation of test results as the biomarkers we tested may have been elevated due to these comorbidities.

Our study has several limitations: (1) Due to the rare incidence of PJI, the sample size of this study is small and we did not acquire enough cases to further analyze the diagnostic performance of biomarkers in different subtypes of PJI; (2) Four cases were excluded because no suitable matches were found, which further limited the sample size of our study; and (3) There were significantly more TKA cases in the PJI group than in the Aseptic group, which may have led to potential bias.

\section{Conclusions}

The level of plasma Fbg and D-dimer was significantly higher in the culture-positive group, compared to the culture-negative group. Plasma Fbg exhibited similar diagnostic performance compared to CRP and ESR. Plasma D-dimer is of limited diagnostic value in PJI. In our study, Fbg and D-dimer did not show better diagnostic performance in any subtypes of PJI. Further studies are required to investigate the difference between serum D-dimer and plasma D-dimer in the arthroplasty population.

\section{Acknowledgements}

This work was supported by the Non-profit Central Research Institute Fund of Chinese Academy of Medical Science. Grant number NWB20204183.

\section{Authors' contributions}

Study design: WWQ. Data analysis : XC, YOW, SB Zhu. Data Collection:, XSW,

JJ JL. Writing: XC. The author(s) read and approved the final manuscript.

\section{Funding}

This work was supported by the Non-profit Central Research Institute Fund of Chinese Academy of Medical Science. Grant number NWB20204183.

\section{Availability of data and materials}

Raw data and materials of this study is deposited in the local server of Peking Union Medical College Hospital for safety and confidentiality purpose. Our data are available upon request for non-commercial research purpose. Please contact the corresponding author at qianww007@163.com for more information.

\section{Declarations}

\section{Ethics approval and consent to participate}

Approval from the Institutional Ethics Committee of Peking Union Medical College Hospital was obtained. The protocol number is S-K1339. For patients who were illiterate and could not sign their names, verbal informed consent from the patients and written informed consent from the patient's guardian who was also present were acquired. Written informed consents were obtained from patients who were literate.

\section{Consent for publication}

Not applicable.

\section{Competing interests}

All authors declare no competing interests.
Received: 29 December 2020 Accepted: 20 April 2021

Published online: 07 May 2021

\section{References}

1. Parvizi J, Tan TL, Goswami K, Higuera C, Della Valle C, Chen AF, Shohat N. The 2018 Definition of Periprosthetic Hip and Knee Infection: An EvidenceBased and Validated Criteria. J Arthroplast. 2018;33(5):1309-14.e1302.

2. Parvizi J, Gehrke T, Chen AF: Proceedings of the International Consensus on Periprosthetic Joint Infection. Bone Joint J2013, 95-b(11):1450-1452.

3. Shahi A, Kheir MM, Tarabichi M, Hosseinzadeh HRS, Tan TL, Parvizi J. Serum D-Dimer Test Is Promising for the Diagnosis of Periprosthetic Joint Infection and Timing of Reimplantation. J Bone Joint Surg Am. 2017;99(17):1419-27.

4. Schindler M, Christofilopoulos P, Wyssa B, Belaieff W, Garzoni C, Bernard L, Lew D, Hoffmeyer P, Uçkay I. Poor performance of microbiological sampling in the prediction of recurrent arthroplasty infection. Int Orthop. 2011;35(5): 647-54

5. Pérez-Prieto D, Portillo ME, Puig-Verdié L, Alier A, Martínez S, Sorlí L, Horcajada JP, Monllau JC. C-reactive protein may misdiagnose prosthetic joint infections, particularly chronic and low-grade infections. Int Orthop. 2017:41(7):1315-9.

6. Deirmengian C, Kardos K, Kilmartin P, Cameron A, Schiller K, Parvizi J. Diagnosing periprosthetic joint infection: has the era of the biomarker arrived? Clin Orthop Relat Res. 2014;472(11):3254-62.

7. Huang J, Zhang Y, Wang Z, Dong Y, Zhao Y, Zheng J, Lian H, Jin Y. The serum level of D-Dimer is not suitable for distinguishing between prosthetic joint infection and aseptic loosening. J Orthop Surg Res. 2019;14(1):407.

8. Li R, Shao HY, Hao LB, Yu BZ, Qu PF, Zhou YX, Chen JY. Plasma Fibrinogen Exhibits Better Performance Than Plasma D-Dimer in the Diagnosis of Periprosthetic Joint Infection: A Multicenter Retrospective Study. J Bone Joint Surg Am. 2019;101(7):613-9.

9. Xiong L, Li S, Dai M. Comparison of D-dimer with CRP and ESR for diagnosis of periprosthetic joint infection. J Orthop Surg Res. 2019;14(1):240.

10. Parvizi J, Gehrke T. Definition of periprosthetic joint infection. J Arthroplast. 2014;29(7):1331.

11. Hansrani V, Khanbhai M, McCollum C. The Diagnosis and Management of Early Deep Vein Thrombosis. Adv Exp Med Biol. 2017;906:23-31.

12. Chung S, Kim JE, Park S, Han KS, Kim HK. Neutrophil and monocyte activation markers have prognostic impact in disseminated intravascular coagulation: in vitro effect of thrombin on monocyte CD163 shedding. Thromb Res. 2011;127(5):450-6.

13. Cicala C, Cirino G. Linkage between inflammation and coagulation: an update on the molecular basis of the crosstalk. Life Sci. 1998;62(20):1817-24.

14. Fu J, Ni M, Chai W, Li X, Hao L, Chen J. Synovial Fluid Viscosity Test is Promising for the Diagnosis of Periprosthetic Joint Infection. J Arthroplast. 2019;34(6):1197-200

15. Qin L, Li F, Gong X, Wang J, Huang W, Hu N. Combined Measurement of DDimer and C-Reactive Protein Levels: Highly Accurate for Diagnosing Chronic Periprosthetic Joint Infection. J Arthroplast. 2020;35(1):229-34.

16. Xu H, Xie J, Huang Q, Lei Y, Zhang S, Pei F. Plasma Fibrin Degradation Product and D-Dimer Are of Limited Value for Diagnosing Periprosthetic Joint Infection. J Arthroplast. 2019;34(10):2454-60.

17. Zhang Q, Dong J, Zhou D, Liu F. Circulating D-Dimer versus Fibrinogen in the Diagnosis of Peri-Prosthetic Joint Infection: A Meta-Analysis. Surg Infect. 2021;22(2):200-10.

18. Chen X, Li H, Zhu S, Wang Y, Qian W. Is D-dimer a reliable biomarker compared to ESR and CRP in the diagnosis of periprosthetic joint infection? Bone Joint Res. 2020;9(10):701-8.

19. Paniccia R, Prisco D, Bandinelli B, Fedi S, Giusti B, Pepe G, Abbate R. Plasma and serum levels of D-dimer and their correlations with other hemostatic parameters in pregnancy. Thromb Res. 2002;105(3):257-62.

20. Gaffney PJ. Standards in fibrinolysis-current status and future challenges. Thromb Haemost. 1995;74(6):1389-97.

21. Wu Y, Xiao YX, Huang TY, Zhang XY, Zhou HB, Zhang XX, Wu YE. What makes D-dimer assays suspicious-heterophilic antibodies? J Clin Lab Anal. 2019;33(2):e22687.

22. Pannu TS, Villa JM, Patel PD, Riesgo AM, Barsoum WK, Higuera CA. The Utility of Serum d-Dimer for the Diagnosis of Periprosthetic Joint Infection in Revision Total Hip and Knee Arthroplasty. J Arthroplast. 2020;35(6):1692-5.

23. Ko YP, Flick MJ. Fibrinogen Is at the Interface of Host Defense and Pathogen Virulence in Staphylococcus aureus Infection. Semin Thromb Hemost. 2016;42(4):408-21. 
24. Dunn DL, Simmons RL. Fibrin in peritonitis. III. The mechanism of bacterial trapping by polymerizing fibrin. Surgery. 1982;92(3):513-9.

25. Bytniewski P, Machała W, Romanowski L, Wiśniewski W, Kosowski K. The dynamics of D-dimer level fluctuation in patients after the cemented and cementless total hip and total knee replacement. J Orthop Surg Res. 2014;9:89.

\section{Publisher's Note}

Springer Nature remains neutral with regard to jurisdictional claims in published maps and institutional affiliations.

Ready to submit your research? Choose BMC and benefit from:

- fast, convenient online submission

- thorough peer review by experienced researchers in your field

- rapid publication on acceptance

- support for research data, including large and complex data types

- gold Open Access which fosters wider collaboration and increased citations

- maximum visibility for your research: over $100 \mathrm{M}$ website views per year

At $\mathrm{BMC}$, research is always in progress.

Learn more biomedcentral.com/submissions 